\title{
Terahertz-based subfemtosecond metrology of relativistic electron beams
}

\author{
R. K. Li, ${ }^{1,}{ }^{*}$ M. C. Hoffmann, ${ }^{1}$ E. A. Nanni, ${ }^{1}$ S. H. Glenzer, ${ }^{1,2}$ M. E. Kozina, ${ }^{1}$ \\ A. M. Lindenberg, ${ }^{2,3,4}$ B. K. Ofori-Okai, ${ }^{1}$ A. H. Reid, ${ }^{1}$ X. Shen, ${ }^{1}$ \\ S. P. Weathersby, ${ }^{1}$ J. Yang, ${ }^{1}$ M. Zajac, ${ }^{4}$ and X. J. Wang ${ }^{1, \dagger}$ \\ ${ }^{1}$ SLAC National Accelerator Laboratory, 2575 Sand Hill Road, Menlo Park, California 94025, USA \\ ${ }^{2}$ PULSE Institute, SLAC National Accelerator Laboratory, Menlo Park, California 94025, USA \\ ${ }^{3}$ SIMES, SLAC National Accelerator Laboratory, Menlo Park, California 94025, USA \\ ${ }^{4}$ Department of Materials Science and Engineering, Stanford University, Stanford, California 94305, USA
}

(Received 7 June 2018; published 7 January 2019)

\begin{abstract}
We demonstrate single-shot temporal characterization of relativistic electron bunches using single-cycle terahertz (THz) field streaking. A transverse deflecting structure consisting of a metal slit enables efficient coupling of the $\mathrm{THz}$ field and electron bunch. The intrinsically stable carrier envelope phase and strong gradient of the $\mathrm{THz}$ pulses allow simultaneous, self-calibrated determination of the time-of-arrival with subfemtosecond precision and bunch duration with single-femtosecond precision, respectively, opening up new opportunities for ultrafast electron diffraction as well as accelerator technologies in general.
\end{abstract}

DOI: 10.1103/PhysRevAccelBeams.22.012803

Photons, electrons, and their interplay are at the heart of photonic devices and modern instruments for ultrafast science [1-10]. Electron beams of the highest intensity and brightness are created via photoemission by short laser pulses and then accelerated and manipulated using $\mathrm{GHz}$ radio-frequency (rf) electromagnetic fields. The electron beams can be used either directly in ultrafast electron diffraction experiments or for producing coherent radiation at $\mathrm{UV}$ to $\mathrm{x}$-ray wavelengths [11-13]. High power $\mathrm{GHz}$ rf sources and structures developed and perfected in past decades have been the workhorse for manipulating electron beams, ranging from compression of $\mathrm{keV}$ electron bunches for table-top ultrafast electron diffraction and imaging [14-17] to acceleration and temporal characterization of $\mathrm{GeV}$ electron beams in kilometer-long x-ray free electron lasers (XFELs). One of the main challenges in ultrafast experiments using electron beams is controlling and characterizing the timing of the probe electron beams relative to optical pump pulses with sufficient precision. An inherent limitation of the $\mathrm{rf}$ approach is the timing synchronization between high power rf sources and pump lasers. Although rf technology allows generation and characterization of few-femtosecond electron beams [18,19], a viable path to

\footnotetext{
*1rk@slac.stanford.edu

wangxj@slac.stanford.edu
}

Published by the American Physical Society under the terms of the Creative Commons Attribution 4.0 International license. Further distribution of this work must maintain attribution to the author(s) and the published article's title, journal citation, and DOI. reduce their timing jitter relative to pump optical pulses to below a few tens of femtosecond does not yet exist.

A promising way to address the timing challenge for pump-probe experiments at a fundamental level, is to manipulate the electron beam using electromagnetic radiation intrinsically synchronized with pump lasers. $\mathrm{THz}$ radiation generated by optical rectification or other nonlinear processes is locked in time relative to the driving laser pulses with subfemtosecond accuracy, and is thus an ideal choice. Manipulation of electron beams using rf or $\mathrm{THz}$ radiation involves introducing a certain amount of energyto-time or momentum-to-time correlation in the beam phase space. Since $\mathrm{THz}$ pulses have 2-3 orders of magnitude higher frequencies than $\mathrm{rf}, \mathrm{THz}$ is much more efficient than rf in shaping the electron beams' temporal characteristics.

Strong-field THz pulses have been used for XUV and $\mathrm{x}$-ray pulse characterization with femtosecond resolution through photoelectron streaking [20-22], extracting eV-level-energy photoelectrons from nanotips and atoms through field emission [23], and THz acceleration [24-26] and manipulation [27-29] of in-vacuum free electrons with higher kinetic energies. In a few recent demonstrations, subrelativistic, $<100 \mathrm{keV}$ kinetic energy electron beams from DC sources were compressed using laser-generated $\mathrm{THz}$ fields to tens of femtoseconds, with their timing jitter stabilized to a few femtoseconds as characterized by $\mathrm{THz}$ streaking [30,31].

There is strong incentive to extend $\mathrm{THz}$ control to electron beams of significantly higher bunch charge and multi-MeV relativistic kinetic energy generated by $\mathrm{rf}$ accelerators while simultaneously achieving sub-fs timing precision. The use of high kinetic energies is very effective in suppressing space charge effects for creating and 


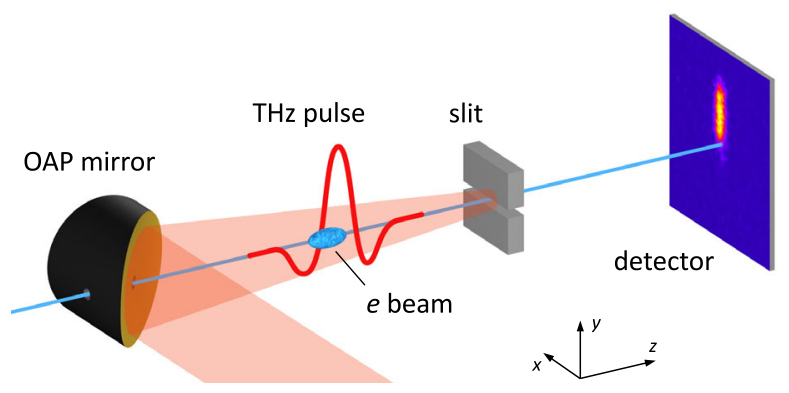

FIG. 1. Schematic of the experimental configuration.

preserving high beam brightness, and thus enabling singleshot measurement of irreversible dynamics in ultrafast diffraction and imaging, as well as studying systems of very low density in the gas phase [32]. However, THz control of bright relativistic electron beams with sub-fs precision has not yet been demonstrated, due to the technical challenges of generating and injecting multi-MeV electron beams from an rf gun with pulse durations and timing jitter both significantly smaller than the $\mathrm{THz}$ wavelength.

In this paper, we report on a method of single-shot determination of the relative time-of-arrival of relativistic electron bunches with subfemtosecond precision and simultaneous single-shot characterization of the bunch length with femtosecond resolution. There is a similar work using laser-generated $\mathrm{THz}$ to characterize the time-ofarrival of relativistic electron beams with a few femtosecond resolution [29]. However, we believe that there are significant differences between our work and Ref. [29] and that our work does indeed represent a major advance in the field of ultrafast electron beams. First, in Ref. [29] the bunch length characterization was carried out using an external rf field and not the $\mathrm{THz}$ pulse itself. Thus, our work is the first demonstration that laser-generated $\mathrm{THz}$ field can characterize $\mathrm{MeV}$ electron beams with a resolution approaching a single femtosecond. Second, the $\mathrm{THz}$ deflecting structures used in the two papers are fundamentally different. We used a parallel plate waveguide (PPWG) structure with a width of $4 \mathrm{~mm}$-roughly one order of magnitude larger than the central wavelength of the $\mathrm{THz}$ field. This wide-band and dispersion-free geometry avoids temporal and spectral distortion of the $\mathrm{THz}$ field along the aperture. With our PPWG structure, the THz field is also transversely uniform and thus can manipulate electron beams with a large acceptance. In contrast, Ref. [29] chose a slit with a width of $250 \mu \mathrm{m}$, which is 2-3 times smaller than the central wavelength of the $\mathrm{THz}$ field, and this structure is highly dispersive with nonuniform fieldenhancement. More detailed comparison will be discussed later in the paper.

Our method is based on deflecting the electrons with a strong single-cycle $\mathrm{THz}$ field using a metal slit structure for optimum transversal momentum transfer. The experiments were conducted at the Accelerator Structure Test Area
(ASTA) facility at SLAC National Accelerator Laboratory [33]. The layout of the experiment is illustrated in Fig. 1.

Quasi-single-cycle THz pulses were generated by optical rectification of $800 \mathrm{~nm}$ laser pulses with up to $16 \mathrm{~mJ}$ energy and $100 \mathrm{fs}$ FWHM duration in a $\mathrm{LiNbO}_{3}$ crystal using the tilted pulse front method [34-36]. The $\mathrm{THz}$ beam was expanded to $\sim 65 \mathrm{~mm} 1 / e^{2}$ diameter using a pair of offaxis parabolic mirrors (OAPs) and transported into the sample vacuum chamber through a polymer window. In vacuum, the THz pulse was focused onto a metal slit using a motorized 90 degree OAP with $76 \mathrm{~mm}$ focal length. A $2.5 \mathrm{~mm}$ diameter hole in the OAP is used to collinearly overlap the relativistic electron beam, as well as an $800 \mathrm{~nm}$ electro-optical sampling (EOS) laser pulse with the $\mathrm{THz}$ pulse. A 50- $\mu$ m thick, 110-cut GaP crystal was used for EO sampling characterization of the THz pulse [37]. The slit structure and the EO crystal were attached to a flat plate parallel to the $x-y$ plane. The plate was mounted on a translation stage which could position either the slit or the EO crystal to the beam axis. The THz polarization was linear and oriented along the $y$ direction. A Spiricon Pyrocam III camera was used to characterize the transverse spot size of the $\mathrm{THz}$ radiation, which was $1.3 \mathrm{~mm}$ FWHM at the focus. The average THz pulse energy was $\sim 20 \mu \mathrm{J}$ measured with an Ophir P3A-THz detector. $\mathrm{THz}$ field strength calculated from EO modulation amplitude in the GaP crystal is $550 \mathrm{kV} / \mathrm{cm}$. This value is in agreement with magnitude of the observed $\mathrm{THz}$ streaking effect and simulation as discussed below.

A relativistic electron beam with $3.1 \mathrm{MeV}$ kinetic energy, $20 \mathrm{fs}$ rms pulse duration, and $3 \mathrm{fC}\left(2 \times 10^{4}\right.$ electrons $)$ bunch charge was generated from a $2.856 \mathrm{GHz}$ photocathode $\mathrm{rf}$ gun operated at $70 \mathrm{MV} / \mathrm{m}$ gradient. The electron beam was collimated by two focusing solenoidal lenses, and injected through the aperture in the parabolic mirror and propagated collinearly with the focused $\mathrm{THz}$ pulse.

When the electron beam and $\mathrm{THz}$ pulse copropagate in vacuum, the electrons experience the Lorentz force $F_{\mathrm{L}}=-e\left(\mathbf{E}_{\mathrm{THz}}+c \beta \times \mathbf{B}_{\mathrm{THz}}\right)$, where $\mathbf{E}_{\mathrm{THz}}$ and $\mathbf{B}_{\mathrm{THz}}$ are the electric and magnetic components of the $\mathrm{THz}, \beta$ is the normalized velocity of electrons, and $e$ and $c$ are the elementary charge and speed of light in vacuum, respectively. In the experiment, the relativistic electron beam has a kinetic energy of $3.1 \mathrm{MeV}$ and travels at $99.0 \%$ of the speed of light. The electric and magnetic force components almost totally counterbalance each other with only $1.0 \%$ residual, illustrating the challenges of $\mathrm{THz}$ control of relativistic electrons compared to nonrelativistic ones for which the net Lorentz force is much stronger. In order to achieve a net momentum transfer and optimum conditions for the streaking measurement, it is necessary to use a nonresonant cavity structure, in our case a metal slit.

The slit, i.e., the transverse deflecting structure, consists of a rectangular slot machined out of $100 \mu \mathrm{m}$ thick $\mathrm{CuAu}$ braze foil. The slit height is $50 \mu \mathrm{m}$ allowing transmission of 

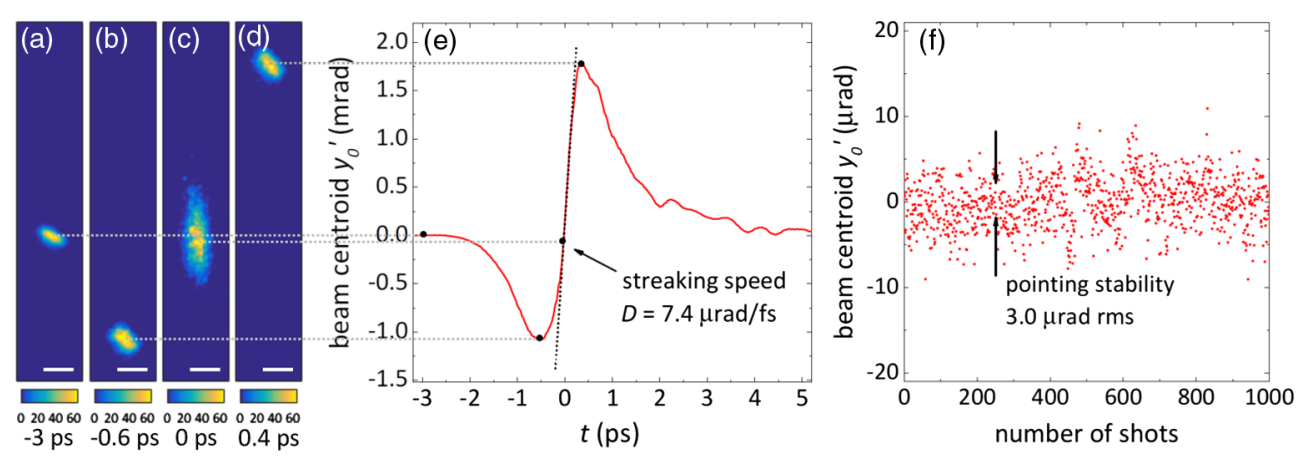

FIG. 2. Experimental data of electron bunch time-of-arrival measurements by $\mathrm{THz}$ streaking. (a)-(d) show raw images of streaked electron bunches at different time delays, indicated by dashed lines in (e) showing the beam centroid versus time delay. The scale bars in (a)-(d) correspond to $1.0 \mathrm{~mm}$ on the detector screen or $0.31 \mathrm{mrad}$ in divergence. (f) Pointing stability of the electron beam when the $\mathrm{THz}$ is off.

most of the focused electron beam. The slit dimension in the transverse plane perpendicular to the electric field polarization is large enough $(4 \mathrm{~mm})$ that the $\mathrm{THz}$ pulse effectively observes a parallel plate rectangular waveguide (PPWG). The structure itself is therefore dispersion free, and the interaction with the electron beam is a result of the impedance mismatch between the slit and the surrounding vacuum.

The driving laser pulses for the electron-beam and $\mathrm{THz}$ generation are split from a common laser pulse, so that the time delay between the $\mathrm{THz}$ and electron beams can be controlled by adjusting the optical path length, leaving the rfinduced timing jitter between $\mathrm{THz}$ and electron pulses as the dominant synchronization uncertainty. The $\mathrm{THz}$ and electron beams are adjusted to overlap spatially and temporally around the $\mathrm{THz}$ focus where the interaction is strongest.

The Lorentz force integrated over the entire interaction region results in a time-dependent transverse momentum kick $\Delta p_{y}$ to the electrons. The value of $\Delta p_{y}$ is determined by the temporal profile of the $\mathrm{THz}$ radiation and the relative time delay between the $\mathrm{THz}$ and electron beams. After the slit, the electron bunch travels freely over a distance of $3.2 \mathrm{~m}$ where the resulting spatial profile is measured by an area detector. Figure 2(a)-2(d) shows unprocessed detector images for single electron bunches at four different time delays. In order to map this spatial information to absolute units of time, we record a calibration curve [Fig. 2(e)] where the average centroid position of electron beam is shown as a function of delay between $\mathrm{THz}$ and photocathode drive laser pulses. One can then map the arrival time of each electron pulse on a shot-by-shot basis onto the time axis using the calibration curve. It is worth to emphasize that this calibration curve alone, which is characterized experimentally, is adequate for unambiguous temporal characterization of electron beams, without the need of additional input such as the $\mathrm{THz}$ profile, the slit properties or field simulations.

The accuracy of the time-of-arrival determination is given by $\sigma_{y 0} / D$, where $\sigma_{y 0}=3.0 \mu \mathrm{rad} \mathrm{rms}$ is the pointing stability of the electron beam when the $\mathrm{THz}$ is not present [Fig. 2(f)], and $D$ is the slope of the calibration curve. With an experimental value of $D=7.4 \mu \mathrm{rad} / \mathrm{fs}$, the timing accuracy is $0.41 \mathrm{fs}$ rms when the electrons arrive around $t=0 \mathrm{ps}$ in Fig. 2(e) where the streaking effect is strongest, and the streaking ramp is linear. Note that the linear portion of the streaking ramp extends about $0.6 \mathrm{ps}$. This time window is significantly wider than the shot-to-shot timeof-arrival jitter of the electron beams, which is $50 \mathrm{fs}$ rms or less in our machine, allowing an unambiguous assignment of arrival time.

While the centroid of the electron bunch provides precise time-of-arrival information, the spatial profile allows simultaneous single-shot determination of the absolute bunch duration with femtosecond resolution. In Fig. 3(a) and 3(b), we show two single-shot snapshots of electron beam profiles with the $\mathrm{THz}$ off and on, respectively. With the position-to-time correlation curve shown in Fig. 2(e) and deconvolution with the nonstreaked beam profile, it is straightforward to convert the streaked beam profile shown in Fig. 3(b) into its temporal distribution. The resolution of the measurement, related to the finite size of the unstreaked beams, is evaluated to be 2.5 fs rms. Further improvement of the resolution can be achieved by reducing the beam size, increasing the $\mathrm{THz}$ strength, and utilizing more efficient interaction structures.

In Fig. 3(d) and 3(f), we show the measured relative-tolaser timing and absolute bunch length of 1000 shots of electron beams recorded over a time period of 50 seconds. We observe both a slow timing drift related to the average phase stability of the rf source relative to the laser, as well as a fast shot-to-shot timing jitter. The slow timing drift, roughly 90 fs over 50 seconds or $\sim 2 \mathrm{fs} / \mathrm{sec}$, can be tracked by a slow feedback and hence $\mathrm{THz}$ streaking time stamping can always be performed in the linear and large-slope region. The shot-to-shot timing jitter is $45.8 \mathrm{fs}$ rms and the bunch length is $21.3 \pm 1.3 \mathrm{fs}$ rms. This information can be used for future pump-probe experiments with femtosecond laser excitation and electron diffraction probe. One possible 

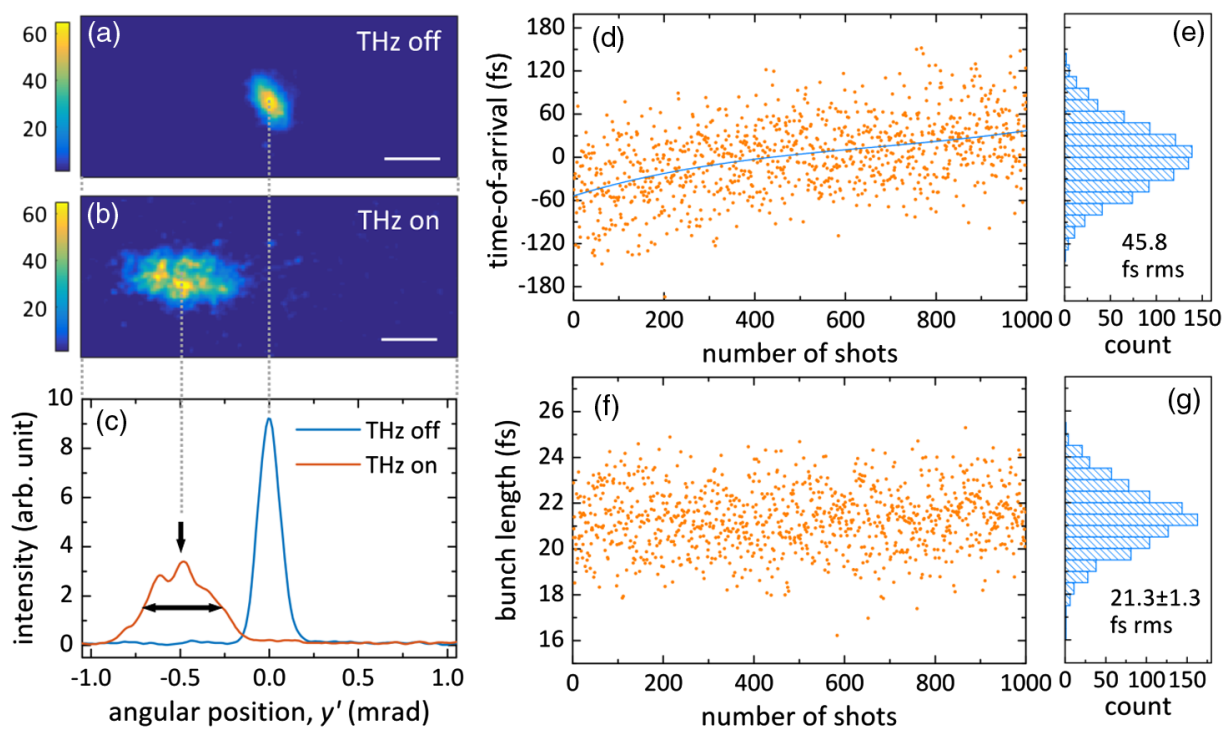

FIG. 3. Experimental data of the relative-to-laser time jitter and the bunch length of the electron beams determined from single-shot $\mathrm{THz}$ streaking images. From (a)-(c) the comparison of single-shot electron beams profiles with the THz off and on, one can determine the relative time-of-arrival [vertical arrow in (c)] and bunch length [horizontal arrow in (c)] of every electron pulse. (a)-(c) have same horizontal axes. The scale bars in (a) and (b) correspond to $1.0 \mathrm{~mm}$ on the detector screen or $0.31 \mathrm{mrad}$ in divergence. (d)-(e) show the time-of-arrival of 1000 electron shots. After removing the slow timing drift, the timing stability is $45.8 \mathrm{fs}$ rms. (f)-(g) show the bunch length for the same 1000 shots after deconvolution. The bunch length is $21.3 \pm 1.3 \mathrm{fs}$ rms.

configuration is to place a $\mathrm{THz}$ slit a few $\mathrm{mm}$ after the sample, and the THz pulse will be introduced at a small angle relative to the electron and pump laser beams to avoid illuminating the sample. The measured time-of-arrival of each electron probe bunch, providing time stamp for each single-shot electron scattering images, can be used to resort the images with respect to the pump laser with subfemtosecond accuracy, and thus significantly improve the overall temporal resolution. The strength of the $\mathrm{THz}$ streaking and hence the timing accuracy will be adjusted to be a fraction of the bunch length of the probe electron beams, so that the contribution of the timing jitter term to the overall temporal resolution can be effectively eliminated, and meanwhile minimizing THz-induced distortion of diffraction patterns. This is conceptually similar to the x-ray-optical crosscorrelation technique for XFELs [38,39]. Real-time information about the bunch duration with high resolution can guide optimization of the electron bunches with additional rf [19] or THz driven devices.

To quantitatively understand the interaction between the relativistic electrons and the THz-induced fields we carried out simulations of the electromagnetic field inside the slit. We use the temporal profile of the $\mathrm{THz}$ as measured by EOS at the focal point in the simulation package HFSS [40]. A Fourier transform of the EOS trace provides the spectral amplitude and phase of the THz pulse. Simulations are performed in the frequency domain from 0.1 to $1.5 \mathrm{THz}$ to cover the relevant spectrum of the input pulse. The simulated spatial volume covered the slit from $\pm 4 \mathrm{~mm}$ in the longitudinal direction and $\pm 2.5 \mathrm{~mm}$ in the transverse direction. A Gaussian $\mathrm{THz}$ beam profile was used to approximate the field distribution entering the slit. The full three-dimensional fields from the frequency domain solver with the correct amplitude and phase were converted back into the time domain where the interaction with electrons is calculated. The measured and simulated angular positions of the electrons at different time delays are shown in Fig. 4(a). The results match remarkably well between -0.3 to $0.3 \mathrm{ps}$ when the streaking slope is steepest. The simulation results show faster changes at other time delays with the discrepancy due to the fact that the EOS of the $\mathrm{THz}$ is inaccurate for very low frequencies, thus limiting the accuracy of our particle motion simulation.

The electric and magnetic components of the Lorentz force experienced by electrons at two different time delays which end up with the maximum and zero integrated angular kicks are shown in Fig. 4(c) and 4(e), respectively. The $100-\mu \mathrm{m}$-thick slit structure is indicated by the two dashed lines. As the $\mathrm{THz}$ and the electrons are far away from the slit, the net Lorentz force is weak. Within a distance of a few $\mathrm{THz}$ wavelengths from the slit, the reflection results in a different enhancement of the $E$ and $B$ fields and introduces a significant phase shift between the two components, which leads to strong net Lorentz forces. The slit effectively behaves as an open terminal on a transmission line allowing for an enhancement of the electric field while requiring a minimum in the magnetic field. The simulations confirm this interpretation showing that although the particles do see kicks from both $E$ and $B$ fields, it is the enhanced electric field on the back face of the slit $(z=0.1 \mathrm{~mm})$ (enhanced by roughly a factor of two compared to the incident field) that results in the net 

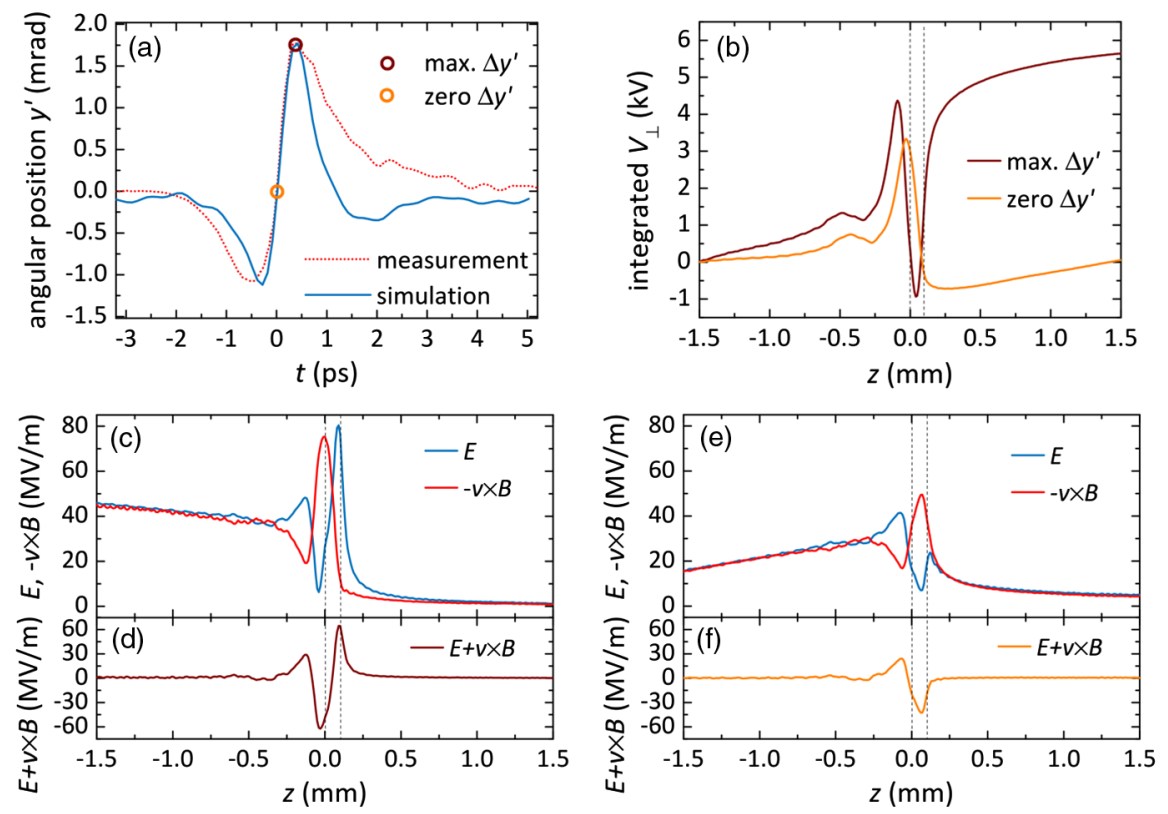

FIG. 4. Simulation results of angular position, integrated transverse voltage, and Lorentz forces experienced by the relativistic electrons. (a) Comparison between the measured and simulated final angular position of the electrons at different time delays. (b)-(f) compare the kinetics of two particles highlighted in (a): "max. $\Delta y^{\prime \prime}$ " with the maximum change in angular position and "zero $\Delta y^{\prime \prime}$ " with the angular motion integrated to zero. The two dashed vertical lines indicate the slit. (b) compares the integrated transverse voltage experienced by the two particles. (c)-(d) show the components and combined Lorentz force experienced by the "max. $\Delta y^{\prime \prime}$ " particle. (e)-(f) show the components and combined Lorentz force experienced by the "zero $\Delta y^{\prime \prime}$ particle.

integrated deflection, as shown in Fig. 4(b). The THz field sampled by the electron beam is quasi-uniform along the horizontal $(x)$ direction since the spot size of Gaussian THz profile is roughly one order of magnitude larger than the spot size of the electron beam. This uniform field distribution over a large transverse extent enabled by the PPWG geometry, compared to the non-uniform distribution in a narrow-band, field-enhancing resonant structure [29], is a highly desired advantage for manipulating electron beams. In contrast, the structure used in Ref. [29] is dispersive, and thus significant ringing (continued periodic deflection of the beam) was observed after the initial excitation with the $\mathrm{THz}$ pulse. Moreover, the enhanced filed is highly nonuniform along the slit direction-with a maximum in the center of the slit and at zero at the edges. This non-uniform field distribution introduces nonlinearities and limits beam quality. Overall, the simulations illustrate the effectiveness of a simple PPWG slit for efficient momentum transfer in THz streaking.

In summary, we demonstrated the simultaneous determination of relative time-of-arrival and bunch duration of a relativistic electron beam by $\mathrm{THz}$ streaking, with a resolution below and approaching a single femtosecond, respectively. The result is a major advance in manipulating high energy electrons using all-optical methods and holds tremendous potential in creating and characterizing isolated electron beams in the subfemtosecond regime [41-44]. A distinct advantage of utilizing laser-generated $\mathrm{THz}$ is that the manipulation of electron beams is intrinsically synchronized to the driving laser, which essentially eliminates the timing-jitter challenge in pump-probe ultrafast electron scattering measurements and external injection in laser-driven accelerators [45-47]. Utilizing such dramatically improved temporal resolution, one can explore the intriguing opportunity of bright electron-beam based spatiotemporal mapping of the $\mathrm{THz}$ electromagnetic fields in metamaterial devices [48] and optically excited wakefields in plasma and dielectric structures with nanometer and subfemtosecond resolutions. With stronger $\mathrm{THz}$ radiation [35] and more efficient interaction structures [26], $\mathrm{THz}$ metrology can be extended to GeV-kinetic-energy electron beams used at XFELs.

The authors gratefully acknowledge the technical support from SLAC Accelerator Directorate, Technology Innovation Directorate and LCLS Laser Science and Technology Division. This work was supported by the U.S. Department of Energy (DOE) under Contract No. DE-AC0276SF00515, the DOE Fusion Energy Sciences under FWP 100182, the DOE Basic Energy Sciences (BES) Accelerator and Detector R\&D program and the SLAC UED/UEM Initiative Program Development Fund. A. M. L. acknowledges support by the DOE, Office of Science, BES, Materials Sciences and Engineering Division. M. C. H. is supported by the DOE, Office of Science, BES, under Award No. 2015-SLAC-100238-Funding.

R. K. L., M.C. H. and E. A. N. contributed equally to this work. 
[1] D. Strickland and G. Mourou, Compression of amplified chirped optical pulses, Opt. Commun. 55, 447 (1985).

[2] G. Mourou and S. Williamson, Picosecond electron diffraction, Appl. Phys. Lett. 41, 44 (1982).

[3] A. Stolow, A. E. Bragg, and D. M. Neumark, Femtosecond time-resolved photoelectron spectroscopy, Chem. Rev. 104, 1719 (2004).

[4] W. E. King, G. H. Campbell, A. Frank, B. Reed, J. F. Schmerge, B. J. Siwick, B. C. Stuart, and P. M. Weber, Ultrafast electron microscopy in materials science, biology, and chemistry, J. Appl. Phys. 97, 111101 (2005).

[5] M. Chergui and A. H. Zewail, Electron and X-ray methods of ultrafast structural dynamics: Advances and applications, Chem. Phys. Chem. 10, 28 (2009).

[6] F. Krausz and M. Ivanov, Attosecond physics, Rev. Mod. Phys. 81, 163 (2009).

[7] A.H. Zewail, Four-dimensional electron microscopy, Science 328, 187 (2010).

[8] G. Sciaini and R.J.D. Miller, Femtosecond electron diffraction: Heralding the era of atomically resolved dynamics, Rep. Prog. Phys. 74, 096101 (2011).

[9] R. J. D. Miller, Femtosecond crystallography with ultrabright electrons and x-rays: Capturing chemistry in action, Science 343, 1108 (2014).

[10] C. Bostedt, S. Boutet, D. M. Fritz, Z. Huang, H. J. Lee, H. T. Lemke, A. Robert, W. F. Schlotter, J. J. Turner, and G. J. Williams, Linac Coherent Light Source: The first five years, Rev. Mod. Phys. 88, 015007 (2016).

[11] P. Emma et al., First lasing and operation of an ångstromwavelength free-electron laser, Nat. Photonics 4, 641 (2010).

[12] T. Ishikawa et al., A compact X-ray free-electron laser emitting in the sub-ångström region, Nat. Photonics 6, 540 (2012).

[13] H.-S. Kang et al., Hard X-ray free-electron laser with femtosecond-scale timing jitter, Nat. Photonics 11, 708 (2017).

[14] T. van Oudheusden, P. L. E. M. Pasmans, S. B. van der Geer, M. J. de Loos, M. J. van der Wiel, and O. J. Luiten, Compression of Subrelativistic Space-Charge-Dominated Electron Bunches for Single-Shot Femtosecond Electron Diffraction, Phys. Rev. Lett. 105, 264801 (2010).

[15] R. P. Chatelain, V. R. Morrison, C. Godbout, and B. J. Siwick, Ultrafast electron diffraction with radio-frequency compressed electron pulses, Appl. Phys. Lett. 101, 081901 (2012).

[16] M. Gao, Y. Jiang, G. H. Kassier, and R. J. D. Miller, Single shot time stamping of ultrabright radio frequency compressed electron pulses, Appl. Phys. Lett. 103, 033503 (2013).

[17] A. Gliserin, M. Walbran, F. Krausz, and P. Baum, Subphonon-period compression of electron pulses for atomic diffraction, Nat. Commun. 6, 8723 (2015).

[18] C. Behrens, F. J. Decker, Y. Ding, V. A. Dolgashev, J. Frisch, Z. Huang, P. Krejcik, H. Loos, A. Lutman, T. J. Maxwell, J. Turner, J. Wang, M. H. Wang, J. Welch, and J. Wu, Few-femtosecond time-resolved measurements of X-ray free-electron lasers, Nat. Commun. 5, 3762 (2014).

[19] J. Maxson, D. Cesar, G. Calmasini, A. Ody, P. Musumeci, and D. Alesini, Direct Measurement of Sub-10 fs Relativistic Electron Beams with Ultralow Emittance, Phys. Rev. Lett. 118, 154802 (2017).
[20] U. Frühling, M. Wieland, M. Gensch, T. Gebert, B. Schütte, M. Krikunova, R. Kalms, F. Budzyn, O. Grimm, J. Rossbach, E. Plönjes, and M. Drescher, Single-shot terahertz-field-driven X-ray streak camera, Nat. Photonics 3, 523 (2009).

[21] I. Grguraš, A. R. Maier, C. Behrens, T. Mazza, T. J. Kelly, P. Radcliffe, S. Düsterer, A. K. Kazansky, N. M. Kabachnik, Th. Tschentscher, J. T. Costello, M. Meyer, M. C. Hoffmann, H. Schlarb, and A. L. Cavalieri, Ultrafast X-ray pulse characterization at free-electron lasers, Nat. Photonics 6, 852 (2012).

[22] M. C. Hoffmann et al., Femtosecond profiling of shaped $\mathrm{X}$-ray pulses, Femtosecond profiling of shaped X-ray pulses, New J. Phys. 20, 033008 (2018).

[23] L. Wimmer, G. Herink, D. R. Solli, S. V. Yalunin, K. E. Echternkamp, and C. Ropers, Terahertz control of nanotip photoemission, Nat. Phys. 10, 432 (2014).

[24] E. A. Nanni, W. R. Huang, K.-H. Hong, K. Ravi, A. Fallahi, G. Moriena, R. J. D. Miller, and F. X. Kärtner, Terahertz-driven linear electron acceleration, Nat. Commun. 6, 8486 (2015).

[25] W. R. Huang, A. Fallahi, X. Wu, H. Cankaya, A.-L. Calendron, K. Ravi, D. Zhang, E. A. Nanni, K.-H. Hong, and F.X. Kärtner, Terahertz-driven, all-optical electron gun, Optica 3, 1209 (2016).

[26] E. Curry, S. Fabbri, J. Maxson, P. Musumeci, and A. Gover, Meter-Scale Terahertz-Driven Acceleration of a Relativistic Beam, Phys. Rev. Lett. 120, 094801 (2018).

[27] J. Fabiańska, G. Kassier, and T. Feurer, Split ring resonator based THz-driven electron streak camera featuring femtosecond resolution, Sci. Rep. 4, 5645 (2014).

[28] E. Curry, S. Fabbri, P. Musumeci, and A. Gover, THzdriven zero-slippage IFEL scheme for phase space manipulation, New J. Phys. 18, 113045 (2016).

[29] L. Zhao et al., Terahertz Streaking of Few-Femtosecond Relativistic Electron Beams, Phys. Rev. X 8, 021061 (2018).

[30] C. Kealhofer, W. Schneider, D. Ehberger, A. Ryabov, F. Krausz, and P. Baum, All-optical control and metrology of electron pulses, Science 352, 429 (2016).

[31] D. Zhang, A. Fallahi, M. Hemmer, X. Wu, M. Fakhari, Y. Hua, H. Cankaya, A.-L. Calendron, L. E. Zapata, N. H. Matlis, and F.X. Kärtner, Segmented terahertz electron accelerator and manipulator (STEAM), Nat. Photonics 12, 336 (2018).

[32] X. J. Wang, D. Xiang, T. K. Kim, and H. Ihee, Potential of femtosecond electron diffraction using near-relativistic electrons from a photocathode rf electron gun, J. Korean Phys. Soc. 48, 583 (2006).

[33] S. P. Weathersby et al., Mega-electron-volt ultrafast electron diffraction at SLAC National Accelerator Laboratory, Rev. Sci. Instrum. 86, 073702 (2015).

[34] J. Hebling, G. Almási, I. Z. Kozma, and J. Kuhl, Velocity matching by pulse front tilting for large-area THz-pulse generation, Opt. Express 10, 1161 (2002).

[35] J. A. Fülöp, L. Pálfalvi, M. C. Hoffmann, and J. Hebling, Towards generation of mJ-level ultrashort $\mathrm{THz}$ pulses by optical rectification, Opt. Express 19, 15090 (2011).

[36] B. K. Ofori-Okai et al., A terahertz pump mega-electronvolt ultrafast electron diffraction probe apparatus at the 
SLAC Accelerator Structure Test Area facility, J. Instrum. 13, P06014 (2018).

[37] Q. Wu and X.-C. Zhang, Free-space electro-optic sampling of terahertz beams, Appl. Phys. Lett. 67, 3523 (1995).

[38] M. Harmand et al., Achieving few-femtosecond timesorting at hard X-ray free-electron lasers, Nat. Photonics 7, 215 (2013).

[39] N. Hartmann et al., Sub-femtosecond precision measurement of relative X-ray arrival time for free-electron lasers, Nat. Photonics 8, 706 (2014).

[40] ANSYS HFSS: 3D Electromagnetic Field Simulator for RF and Wireless Design, https://www.ansys.com/products/ electronics/ansys-hfss (2018).

[41] C. M. S. Sears, E. Colby, R. Ischebeck, C. McGuinness, J. Nelson, R. Noble, R. H. Siemann, J. Spencer, D. Walz, T. Plettner, and R. L. Byer, Production and characterization of attosecond electron bunch trains, Phys. Rev. ST Accel. Beams 11, 061301 (2008).

[42] K. E. Priebe, C. Rathje, S. V. Yalunin, T. Hohage, A. Feist, S. Schäfer, and C. Ropers, Attosecond electron pulse trains and quantum state reconstruction in ultrafast transmission electron microscopy, Nat. Photonics 11, 793 (2017).

[43] M. Th. Hassan, J. S. Baskin, B. Liao, and A. H. Zewail, High-temporal-resolution electron microscopy for imaging ultrafast electron dynamics, Nat. Photonics 11, 425 (2017).

[44] Y. Morimoto and P. Baum, Diffraction and microscopy with attosecond electron pulse trains, Nat. Phys. 14, 252 (2018).

[45] E. Esarey, C. B. Schroeder, and W. P. Leemans, Physics of laser-driven plasma-based electron accelerators, Rev. Mod. Phys. 81, 1229 (2009).

[46] V. Malka, J. Faure, Y. A. Gauduel, E. Lefebvre, A. Rousse, and K. T. Phuoc, Principles and applications of compact laser-plasma accelerators, Nat. Phys. 4, 447 (2008).

[47] R. J. England et al., Dielectric laser accelerators, Rev. Mod. Phys. 86, 1337 (2014).

[48] Y. Zhu et al., Structural imaging of nanoscale phonon transport in ferroelectrics excited by metamaterial-enhanced terahertz fields, Phys. Rev. Mater. 1, 060601 (2017). 